\title{
Adult Tumors of Soft Tissue, Bone, and Bone Marrow: What the Clinician Wants to Know
}

\author{
Hillary W. Garner and Mark D. Murphey
}

\section{Learning Objectives}

- To gain knowledge and understanding of what clinicians want to know from imaging of soft tissue, bone, and bone marrow tumors.

- To understand how the key imaging features of tumors impact their diagnosis, staging, and treatment options.

- To illustrate examples of report templates for the comprehensive and clear reporting of imaging studies obtained for evaluation of soft tissue, bone, and bone marrow tumors.

\subsection{Introduction}

The function of imaging in the setting of a soft tissue, bone, or bone marrow tumor hinges on the capacity to accurately characterize, localize, and quantitate the abnormality. A radiologist's interpretation and reporting of these key imaging findings allow for a differential diagnosis, can add specificity and confidence in diagnosis, and allow for a determination regarding the need for biopsy and a shared platform from which the clinical team and patient can discuss further diagnostic management and an initial treatment plan. In addition, the appropriate characterization of a tumor can often provide insight into the potential staging of the tumor and guide the need for additional imaging prior to biopsy.

H. W. Garner $(\bowtie)$

Department of Radiology, Mayo Clinic, Jacksonville, FL, USA

e-mail: garner.hillary@mayo.edu

M. D. Murphey

American Institute for Radiologic Pathology,

Silver Spring, MD, USA

Department of Radiology and Nuclear Medicine, Uniformed Services University of the Health Sciences, Bethesda, MD, USA e-mail: mmurphey@acr.org
Adult tumors of soft tissue, bone, and bone marrow encompass a broad range of histopathologies with varying immunohistochemical and molecular properties, but the tenets of imaging for these tumors and their value to clinicians and patients are consistent regardless of tumor type. Demonstrating an understanding of the relevance and influence of the patient's history and addressing the important imaging features of a tumor in a consistent and concise manner in the radiology report elevate the perceived worth of the radiologist's input into patient care. In this chapter, we focus on magnetic resonance imaging (MRI) findings and provide guidance on what clinicians want to know and how to report this information using a clear and uniform approach.

\subsection{Soft Tissue Tumors}

Although the majority of soft tissue tumors (STT) are benign, the morbidity and mortality associated with soft tissue sarcoma (STS) are significant and warrant that radiologists have a fundamental knowledge of their imaging presentations and treatment methods and goals. Radiographs, ultrasound, computed tomography (CT), and positron emission tomography (PET)/CT can all often provide useful information for differential diagnosis of a STT. The purpose of radiographs for the evaluation of STT is often underestimated, but they can provide highly valuable information, such as the identification and characterization of associated mineralization and osseous lesion origin and the identification of underlying cortical involvement, which can influence the differential diagnosis. Therefore, radiographs should be routinely performed during the workup of STT and are an appropriate initial imaging study. For superficial STT, either radiographs or ultrasound is appropriate initially [1]. If the STT requires further characterization after radiographic or ultrasound evaluation, MRI is the imaging technique of choice [2-4] as it often allows for a limited differential diagnosis. For example, the most common myxoid lesions, soft tissue myxoma, 
myxoid liposarcoma, and myxofibrosarcoma (Fig. 10.1), can usually be distinguished by their imaging characteristics in the vast majority of cases on MRI [5-7]. An understanding of the relevance of the MRI features favoring a benign versus malignant diagnosis is paramount to making a determination of radiology-pathology concordance or discordance and making appropriate recommendations for next steps in management. For STS in particular, the key MRI features that help predict its behavior and potentially impact surgical management are the tumor's anatomical site (intramuscular, intermuscular, subcutaneous, intraarticular, extensive/multifocal), size, depth, margin, and morphological/enhancement complexity. Specific locations can also improve diagnostic accuracy, such as deep to the scapular tip for elastofibroma and hand/foot for fibromatosis. Reporting of associated inflammatory edema-like signal or hemorrhage in the surrounding soft tissues or associated fascial enhancement extending from the tumor (fascial tails) is also crucial because these areas can contain tumor cells and needs to be addressed in the treatment plan to maximize local control.

Several recent studies have incorporated the numerous

\section{Key Point}

- Knowledge of the key imaging features that predict tumor behavior is critical to providing a comprehensive imaging report.

elemental constituents of these key MRI features into MRI radiomics models in an effort to better grade [8] and stage [9] STS as well as predict specific histopathology [4], treatment response [10], and overall survival [11]. Therefore, each of these key MRI features should be specifically described in the radiology report. Of note, in the setting of an aggressiveappearing or otherwise indeterminate STT, it behooves radiologists, particularly those practicing outside the tertiary care setting, to clearly and specifically recommend in the final impression of the imaging report that the patient be referred to an orthopedic oncologist prior to biopsy. An

Fig. 10.1 Examples of common pathologically proven myxoid lesions on MRI. Coronal T1-weighted (a), coronal T2-weighted fat-saturated (b), and coronal T1-weighted fat-saturated post-contrast (c) MR images of the proximal right thigh demonstrating a $<5 \mathrm{~cm}$ circumscribed intramuscular mass with homogeneous T1 signal isointense to muscle and a thin rim of perilesional fat (arrowheads in a), homogeneous extremely bright T2 signal with perilesional edema at the superior and inferior margins (arrows in b), and whorl-like areas of mild enhancement internally. There is no enhancement extending away from the mass. These MRI features are most compatible with a soft tissue myxoma. Coronal T1-weighted (d), coronal T2-weighted fat-saturated (e), and example of a structured MRI report for a soft tissue sarcoma is provided in Fig. 10.2.

In addition to an appreciation of the importance of the key MRI features, an understanding of the staging methods for STS is relevant. The most commonly used staging system for almost all histological subtypes of STS is the American Joint Committee on Cancer (AJCC) staging system [12], which incorporates extent of tumor $(\mathrm{T})$, lymph node status $(\mathrm{N})$, presence of metastasis $(M)$, and tumor grade $(\mathrm{G})$. In the most recent 8th edition of the AJCC staging system published in 2017 [13], the anatomical site of STS became a more relevant staging parameter, with different staging systems now employed based on whether the STS is located in the trunk/extremity, retroperitoneum, head and neck, or thoracic/abdominal visceral organs $[12,13]$. In the musculoskeletal radiology realm, we are primarily involved in the assessment of STS of the trunk/extremity, so specific knowledge of the staging system for this anatomical site is useful. The tumor grade in the AJCC staging system is based on the French Federation of Cancer Centers (FNCLCC) grading system, which includes tumor differentiation, presence of necrosis, and mitotic count in its analysis. Radiologists can provide insight on the histological grade of a STS by identifying and reporting necrosis during image interpretation and including areas of necrosis within biopsy specimens. Of note, a few aspects of the AJCC trunk/extremity staging system are controversial, including the designation of regional nodal metastasis as stage IV disease, the exclusion of tumor depth as an independent prognostic factor, and the division of tumors greater than $10 \mathrm{~cm}$ into two separate T categories rather than just one category $(\mathrm{T} 3,>10 \mathrm{~cm} \leq 15 \mathrm{~cm}$; $\mathrm{T} 4$, $>15 \mathrm{~cm}$ ) [14]. Given these controversies and the broad nonuniformity in STS types and behaviors, tumor genomics likely plays a greater role, if not a central role, in future prognostication. In the interim, clinicians rely on clinical examination and the information gleaned from the MRI interpretation to plan treatment.

The mainstay of treatment for STS is wide surgical resection with the primary goal being a balance between achieving local control and preserving patient function. Additional MRI features that are essential to report in an effort to help surgeons best achieve this balance, particularly in the setting coronal T1-weighted fat-saturated post-contrast (f) MR images of the proximal right thigh demonstrating a $>5 \mathrm{~cm}$ fat-containing heterogeneous mass with irregular perilesional edema and enhancement. These MRI features are most compatible with a myxoid liposarcoma. Coronal T1-weighted (g), coronal T2-weighted fat-saturated (h), and coronal T1-weighted fat-saturated post-contrast (i) MR images of the proximal right thigh demonstrating a $>5 \mathrm{~cm}$ heterogeneous mass avid irregular peripheral enhancement, central necrosis, and irregular perilesional edema and enhancement. These MRI features are most compatible with a myxofibrosarcoma 

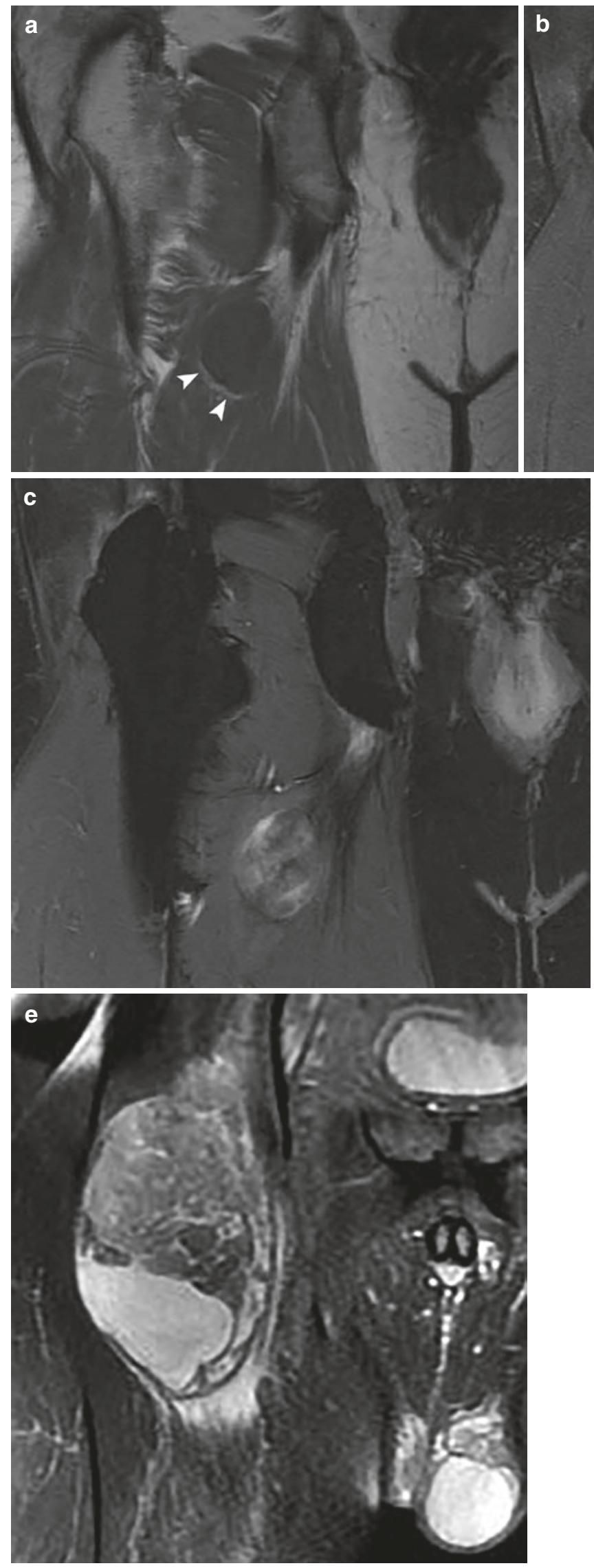
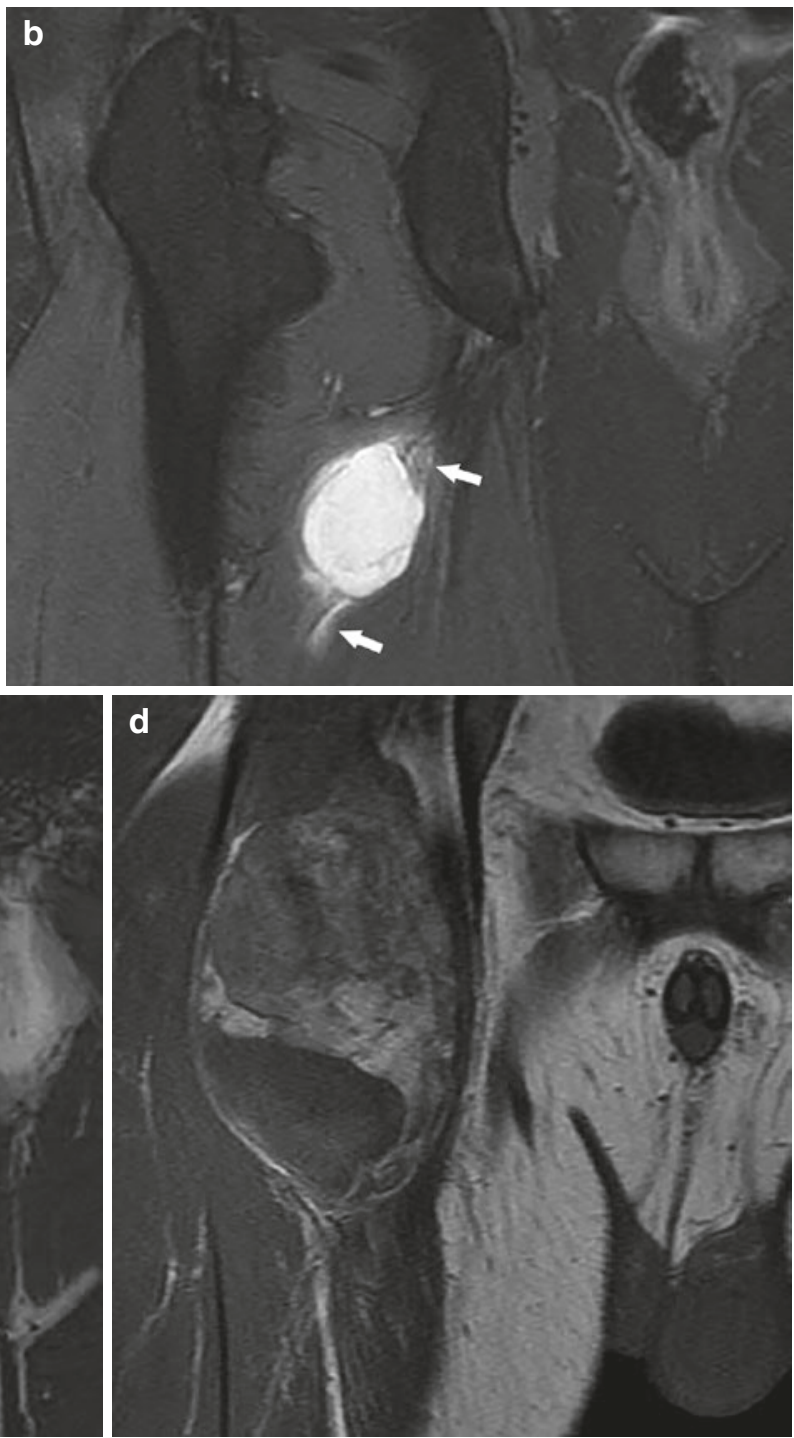

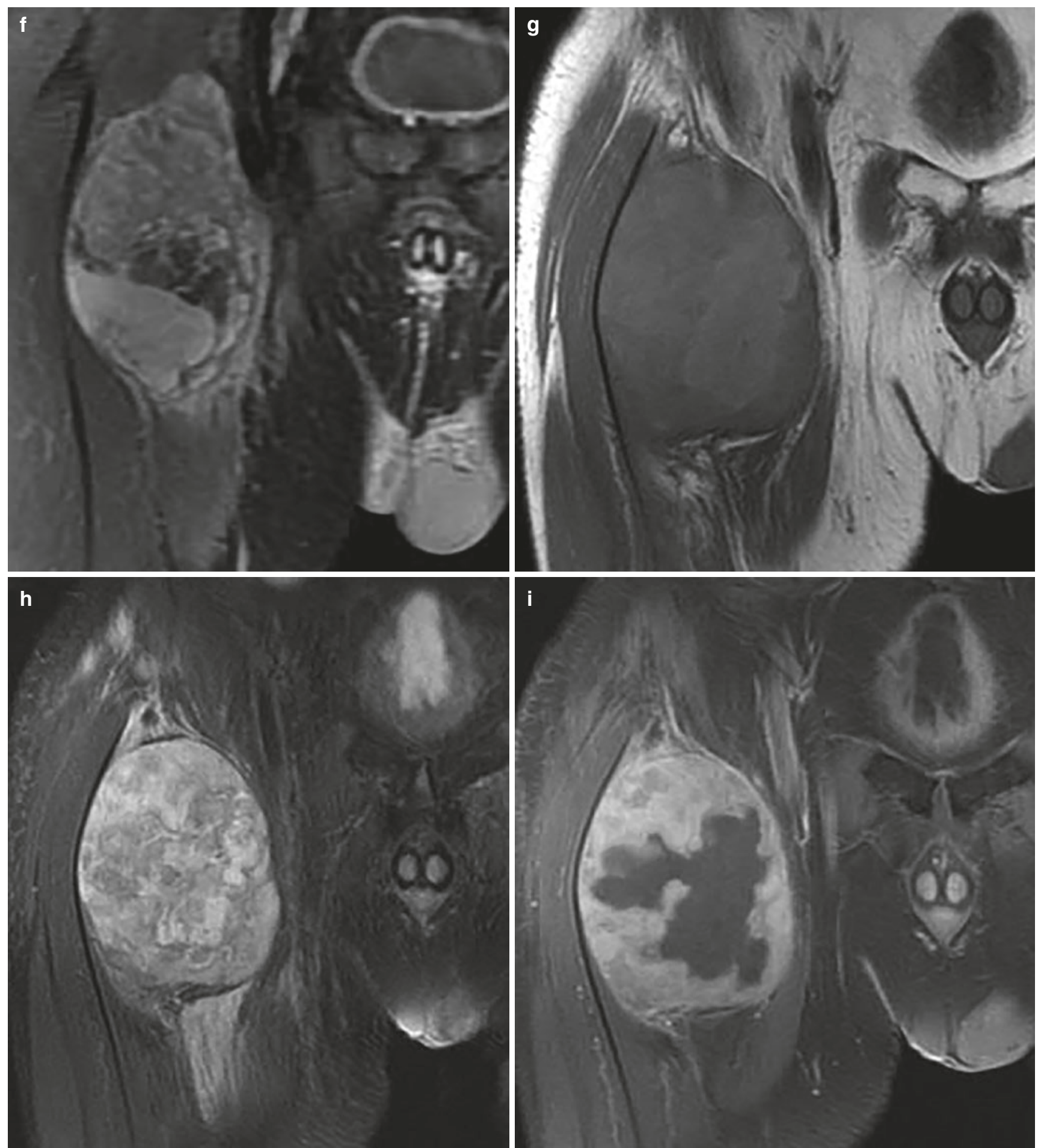

Fig. 10.1 (continued)

of an aggressive-appearing STT, are the presence or absence of muscular compartmental breaching, neurovascular involvement (Fig. 10.3), organ invasion, or bone invasion. This information often dictates the treatment plan, including whether to pursue neoadjuvant therapies prior to resection or to proceed with primary surgical intervention. In the case of neurovascular or organ involvement, the orthopedic oncol- ogy team requires operative assistance by other surgical specialties. Furthermore, if the STT is situated within an anatomically complex region such as the pelvis, the need for three-dimensional (3D) printing for surgical planning can be anticipated.

Another crucial element to the report which often receives less attention from radiologists is a description of the 
EXAM: MRI [ANATOMICAL SITE] WO + W/CON

Technique: Multiplanar multisequence MR imaging of the [anatomical site] was performed before and after the uneventful intravenous administration of $[\mathrm{x}] \mathrm{mL}$ [contrast agent].

History: xx-year-old man/woman presented to an outside physician 1 month ago for evaluation of a slowly enlarging painless mass at the anterior aspect of the [anatomical site] which was first noticed approximately 2 years ago. Outside ultrasound demonstrated a solid vascular mass that was reported as worrisome for malignancy. The patient has no other health concerns.

Comparisons: Radiographs of the [anatomical site] obtained earlier today.

FINDINGS:

Mass characteristics:

Location:

Anatomical site:

Tissue depth/location (subcutaneous, fascial,intra-/intermuscular,intraarticular, multifocal):

Size:

Margin (circumscribed/irregular):

Morphology and T1/T2 signal characteristics:

Enhancement characteristics (avid/heterogeneous/central/peripheral):

Most aggressive portion for biopsy target:

Necrosis (None vs $<50 \%$ vs $>50 \%$ ):

Radiographic appearance (mineralization/cortical involvement):

Sonographic appearance (solid vs cystic/echogenicity/vascularity):

Status of surrounding tissues:

Edema (peritumoral/fascial):

Hemorrhage:

Neurovascular involvement:

Bone involvement:

Other:

Other findings:

Staging information:

Longest diameter of tumor $(\mathrm{T})$ :

Lymph node (L): may be unknown at time of MRI; report expected date of staging CT

Metastasis (M): may be unknown at time of MRI; report expected date of staging CT

Necrosis:

FINAL IMPRESSION:

Fig. 10.2 Example of a structured MRI report for soft tissue sarcoma

patient's history. Accurately and thoroughly addressing the patient's history relevant to the STT directly in the report, particularly in the setting of treated soft tissue sarcoma, gives the referring clinician and the patient confidence in the care that the radiologist is providing. For example, if the patient has a history of an undifferentiated pleomorphic sarcoma, specifically reporting in chronological order the date of initial biopsy diagnosis, the histologic grade, the incorporation of radiation with dates of initiation and conclusion and total dosage, the incorporation of chemotherapy, and the date of any surgeries, whether non-oncologic or oncologic is extremely helpful for bringing together a common language for all involved in the patient's care. Also, a key piece of information that should be sought when interpreting followup MRI after STS resection is the surgical margin status as it is the primary influence on risk of local recurrence [15]. 


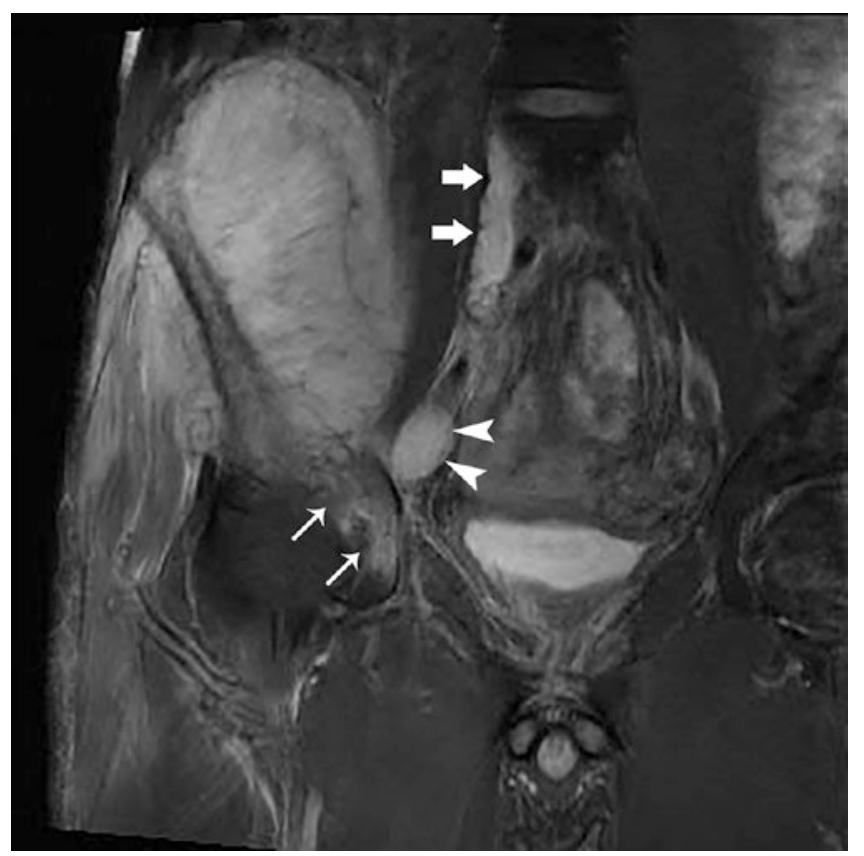

Fig. 10.3 A 36-year-old man with osteosarcoma of the right ilium. Coronal T2-weighted fat-saturated image of the pelvis demonstrates the large primary tumor with invasion into the right hip joint (skinny arrows), a right external iliac lymph node metastasis (arrowheads), and tumor thrombus in the right common iliac vein (fat arrows)

Fortunately, much of this information is now available in the electronic medical record. Other alternatives to obtaining this information could be providing a questionnaire to the patient at the time of check-in for imaging or contacting the patient directly.

\section{Key Point}

- Inclusion of a detailed patient history in the imaging report confirms to the patient and the other members of the healthcare team the investment of the radiologist in helping maximize patient care and outcome.

\subsection{Bone Tumors}

Similar principles guiding the imaging reporting of STT can be applied to bone tumors. Although radiographs are highly valuable in the setting of a STT even when negative, in the setting of extremity bone tumors, accurate characterization of an extremity bone tumor relies heavily on the radiographic appearance. Therefore, radiographs in at least two orthogonal planes are imperative and should be employed as the initial imaging modality. Given that the location of a bone lesion in combination with the patient age allows for a limited differential of two or three diagnoses in most cases, the radio- graphic report should initially include a clear description of the specific location within the bone, whether epiphyseal, metaphyseal, or diaphyseal and whether intramedullary, cortical, or surface. Other key features to analyze and report are the size, zone of transition (narrow or wide), pattern of bone destruction (geographic, moth-eaten, permeative), periosteal reaction (aggressive or nonaggressive), and internal characteristics of the lesion (lucent/lytic, sclerotic, mixed, osteoid/ chondroid matrix). For indeterminate or aggressive-appearing lesions, it is vital to also report on the status of the cortex and soft tissues and assess for risk of pathologic fracture.

\section{Key Point}

- Radiographic evaluation of a bone tumor is imperative. MRI interpretation of a bone lesion without comparison to the corresponding radiographs raises the risk of misdiagnosis and mistreatment.

When radiographs confirm the presence of an aggressive or indeterminate bone lesion in the extremity or pelvis, the modality of choice for further imaging evaluation is MRI, which should be protocoled to include sequences of the entire compartment with the associated proximal and distal joints [16]. Of note, the corresponding radiographs should always be reviewed concurrently during the MRI interpretation, and this direct correlation should be stated in the report. For non-extremity bone lesions, CT is typically used as the primary imaging modality [16], but MRI is also advantageous as a subsequent test as it can better define tissue planes between tumor and surrounding structures. The same features of the lesion described radiographically should similarly be addressed in the MRI and/or CT report. Additionally, the morphological/enhancement complexity can be better defined with these advanced modalities. Other highly important potential findings to identify and describe include physeal transgression, articular invasion, and soft tissue/organ/ neurovascular involvement (Fig. 10.3). Each of these features has implications for diagnosis, staging, biopsy planning, and treatment planning. Therefore, as stated above for STT, radiologists in a non-tertiary care setting can best serve their patients by clearly and specifically recommending in the final impression of the report a referral to an orthopedic oncologist prior to biopsy.

\section{Key Point}

- Patients with high-risk musculoskeletal tumors should only undergo biopsy following consultation with the treating orthopedic oncologist. 
Similar to STS, there are controversies surrounding the available staging systems for bone sarcoma, which include the AJCC 8th edition, Musculoskeletal Tumor Society, and Vanderbilt staging systems. In particular, these staging systems have variable accuracies for predicting overall survival in the three most common types of primary bone sarcoma, which are osteosarcoma, Ewing sarcoma, and chondrosarcoma $[17,18]$. However, radiologists can benefit from the knowledge that histologic grade and the presence and location of metastatic disease are key factors for staging in all of these lesions. Although combining these three most common bone sarcoma types into one staging system simplifies the clinical approach, it may be a disservice to patients who would more likely benefit from individualized prognostic information. Therefore, a greater focus on tumor-type specific genomics is expected to play more of a role in the staging of bone sarcomas in the future. As radiologists, keeping up to date as the staging systems for these tumors evolve over time is important to maintain a common multidisciplinary language.

MRI or CT reports obtained for evaluation of a bone tumor need to include a thorough description of all relevant patient history associated with the bone tumor, particularly in the post-treatment setting. For example, the general treatment strategies for osteosarcoma and Ewing sarcoma incorporate both neoadjuvant and adjuvant chemotherapy, but radiation is usually reserved for anatomical locations where complete surgical resection is difficult or impossible [16]. Therefore, the knowledge that a patient with osteosarcoma or Ewing sarcoma was treated with radiation is helpful in that it alerts the radiologist to the potential of a higher risk of local recurrence. An example of a structured report template for bone sarcoma following treatment is provided in Fig. 10.4.

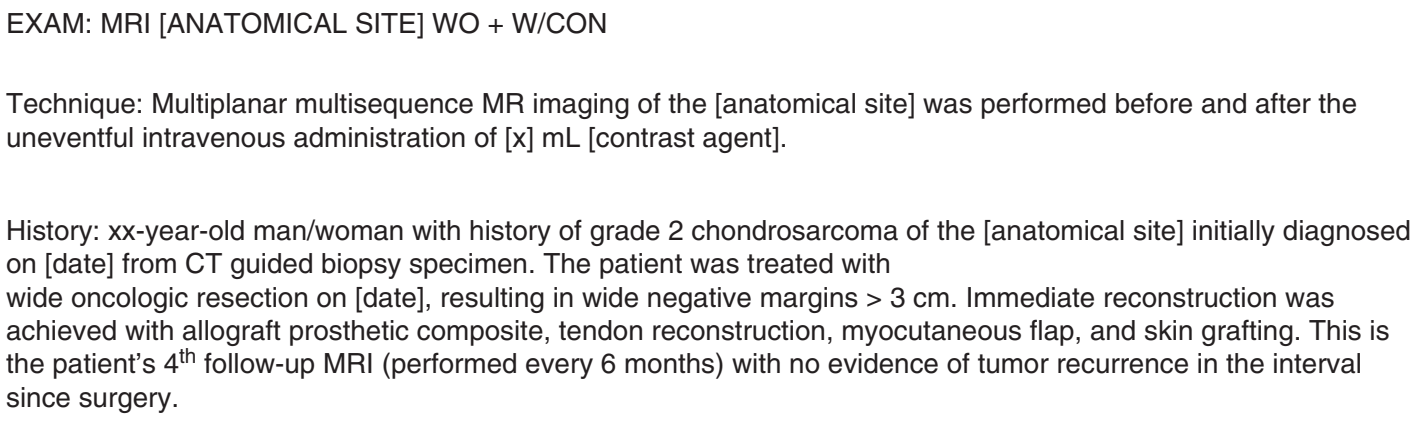

FINAL IMPRESSION:

Fig. 10.4 Example of a structured MRI report for post-treatment follow-up of bone sarcoma 


\subsection{Bone Marrow Tumors}

Primary bone marrow tumors (BMT) include myeloma, leukemia, and lymphoma. Radiographs, CT, PET/CT, and MRI are all used in the evaluation of these tumors. As with bone tumors, radiographs are the most appropriate initial imaging test when the clinical presentation is that of localized unremitting atraumatic bone pain. However, if the clinical presentation of a BMT is instead based on laboratory abnormalities, the historical practice of using skeletal survey for initial evaluation is now considered suboptimal. Currently, low-dose CT, PET/CT, or whole-body MRI are considered acceptable initial imaging modalities today. The primary advantages of low-dose CT are that it is more sensitive than skeletal survey and has short acquisition time and wide availability. However, CT relies on the presence of trabecular and/ or cortical destruction for detection of focal disease [19]. PET/CT can also detect focal bone lesions and has been used for assessment of treatment response and for monitoring, but its sensitivity is lower than that of MRI. Comparatively, whole-body MRI with diffusion weighted imaging is the most sensitive for BMT and allows for the earliest detection of focal disease in the form of cellular infiltration, even before trabecular or cortical destruction [19]. Unfortunately, there remain barriers to whole-body MR's wide implementation into routine clinical practice due to its cost, acquisition time, and challenges with billing and reimbursement [20]. If whole-body MRI is not available, MRI of the spine and pelvis is considered a suitable alternative [19].

When assessing BMT with imaging, the key elements of disease to report are the disease distribution (focal or diffuse), associated soft tissue components, nodal/organ involvement, and the presence of fracture or predicted fracture risk. In regard to focal disease, the International Myeloma Working Group has provided guidance on size measurements using thresholds of 5 and $10 \mathrm{~mm}$. For lesions $\leq 5 \mathrm{~mm}$, documentation of the abnormality is recommended to facilitate continued surveillance, but an exact measurement is not necessary. Lesions that are $>5 \mathrm{~mm}$ but $<10 \mathrm{~mm}$ should be interpreted as unequivocally active myeloma, but again an exact measurement is not necessary [19]. Focal lesions larger than $10 \mathrm{~mm}$ should be reported with associated measurements. If the imaging findings are instead indicative of diffuse disease, this should be identified in the report. In addition, analysis of burden of disease in the posteromedial aspects of the ilia, which are the most commonly used sites for bone marrow aspiration and biopsy, would be helpful to ensure optimal tissue sampling of the most abnormal area or to suggest an alternate site. Reporting of ADC values is also recommended, but only for lesions where diffusion weighted imaging demonstrates water signal. Radiologists can benefit their referring clinicians and patients by following the structured reporting recommen- dations provided by the Myeloma Response Assessment and Diagnosis System (MY-RADS), which was created under the guidance of the International Myeloma Working Group and the National Institute for Clinical Excellence in the United Kingdom [19]. Although a similar system has not been specifically devised for other BMT such as leukemia or lymphoma, a similar approach to image analysis and reporting is recommended.

\section{Key Point}

- The use of structured reporting in the setting of myeloma allows for clear and consistent communication between the radiologist and other members of the healthcare team.

\subsection{Concluding Remarks}

In this review, we have highlighted what clinicians want to know from the imaging of soft tissue, bone, and bone marrow tumors and how the key imaging features influence their diagnosis, staging, and treatment. We have stressed the importance and value of providing a detailed patient history in the imaging report and of explicitly stating the need for referral to an orthopedic oncologist if a sarcoma is suspected. An accurate and quality imaging report that demonstrates investment in the patient's care can elevate the perceived worth of radiologists to the healthcare team.

\section{Take Home Messages}

- Clearly and consistently report the key imaging features that may impact the diagnosis, staging, and treatment of soft tissue, bone, and bone marrow tumors.

- Provide a detailed patient history regarding the tumor in the imaging report.

- Do not hesitate to recommend referral to an orthopedic oncologist in the final impression of the report if an aggressive tumor is suspected.

\section{References}

1. Kransdorf MJ, Murphey MD, Wessell DE, et al. American College of Radiology. ACR Appropriateness Criteria ${ }^{\circledR}$ : soft-tissue masses. 2020. Available at: https://acsearch.acr.org/docs/69434/Narrative/. Accessed 30 Sept 2020.

2. Kransdorf MJ, Murphey MD. Radiologic evaluation of softtissue masses: a current perspective. AJR Am J Roentgenol. 2000;175:575-87. 
3. Manaster BJ. Soft-tissue masses: optimal imaging protocol and reporting. AJR Am J Roentgenol. 2013;201:505-14.

4. Zhang Y, Zhu Y, Shi X, et al. Soft tissue sarcomas: preoperative predictive histopathological grading based on radiomics of MRI. Acad Radiol. 2019;26(9):1262-8.

5. Murphey MD, McRae GA, Fanburg-Smith JC, Temple HT, Levine AM, Aboulafia AJ. Imaging of soft-tissue myxoma with emphasis on CT and MR and comparison of radiologic and pathologic findings. Radiology. 2002;225(1):215-24.

6. Murphey MD, Acara LK, Fanburg-Smith JC. From the archives of the AFIP: imaging of musculoskeletal liposarcoma with radiologicpathologic correlation. Radiographics. 2005;25(5):1371-95.

7. Baheti AD, Tirumani SH, Rosenthal MH, et al. Myxoid soft-tissue neoplasms: comprehensive update of the taxonomy and MRI features. AJR Am J Roentgenol. 2015;204(2):374-85.

8. Peeken JC, Spraker MB, Knebel C, et al. Tumor grading of soft tissue sarcomas using MRI-based radiomics. EBioMedicine. 2019;48:332-40.

9. Vallières M, Freeman CR, Skamene SR, El Naqa I. A radiomics model from joint FDGPET and MRI texture features for the prediction of lung metastases in soft-tissue sarcomas of the extremities. Phys Med Biol. 2015;60:5471-96.

10. Spraker MB, Wootton LS, Hippe DS, et al. MRI radiomic features are independently associated with overall survival in soft tissue sarcoma. Adv Radiat Oncol. 2019;4:413-21.

11. Crombé A, Périer C, Kind M, et al. T2-based MRI Delta-radiomics improve response prediction in soft-tissue sarcomas treated by neoadjuvant chemotherapy. J Magn Reson Imaging. 2019;50:497-510.

12. Gilbert NF, Cannon CP, Lin PP, Lewis VO. Soft-tissue sarcoma. J Am Acad Orthop Surg. 2009;17(1):40-7.
13. Amin MB, Edge S, Greene F, et al., editors. AJCC cancer staging manual. 8th ed. Cham: Springer International Publishing; 2017.

14. Cates JMM. The AJCC 8th edition staging system for soft tissue sarcoma of the extremities or trunk: a cohort study of the SEER database. J Natl Compr Cancer Netw. 2018;16(2):144-52.

15. Spolverato G, Callegaro D, Gronchi A. Defining which patients are at high risk for recurrence of soft tissue sarcoma. Curr Treat Options Oncol. 2020;21(56):1-18.

\section{Bone Tumors}

16. Casali PG, Bielack S, Abecassis N, et al. Bone sarcomas: ESMOPaedCan-EURACAN clinical practice guidelines for diagnosis, treatment and follow-up. Ann Oncol. 2018;29(Suppl 4):iv79-95.

17. Compton ML, Cates JMM. Evidence-based tumor staging of skeletal chondrosarcoma. Am J Surg Pathol. 2020;44(1):111-9.

18. Cates JMM. Simple staging system for osteosarcoma performs equivalently to the AJCC and MSTS systems. J Orthop Res. 2018;36(10):2802-8.

19. Messiou C, Hillengass J, Delorme S, et al. Guidelines for acquisition, interpretation, and reporting of whole-body MRI in myeloma: myeloma response assessment and diagnosis system (MY-RADS). Radiology. 2019;291(1):5-13.

20. Feldhaus JM, Garner HW, Wessell DE. Society of skeletal radiology member utilization and performance of whole-body MRI in adults. Skelet Radiol. 2020;49(11):1731-6.

Open Access This chapter is licensed under the terms of the Creative Commons Attribution 4.0 International License (http://creativecommons. org/licenses/by/4.0/), which permits use, sharing, adaptation, distribution and reproduction in any medium or format, as long as you give appropriate credit to the original author(s) and the source, provide a link to the Creative Commons license and indicate if changes were made.

The images or other third party material in this chapter are included in the chapter's Creative Commons license, unless indicated otherwise in a credit line to the material. If material is not included in the chapter's Creative Commons license and your intended use is not permitted by statutory regulation or exceeds the permitted use, you will need to obtain permission directly from the copyright holder. 